www.jmscr.igmpublication.org

Impact Factor 5.84

Index Copernicus Value: 83.27

ISSN (e)-2347-176x ISSN (p) 2455-0450

crossref DOI: _https://dx.doi.org/10.18535/jmscr/v5i5.201

Journal Of Medical Science And Clinical Research

IGM Publication

An Official Publication of IGM Publication

\title{
Hydroxyzine induced Paroxysmal Supraventricular Tachycardia in a Neonate- an Extremely Rare Case
}

\author{
Authors \\ Dr Sandhya Chauhan ${ }^{1}$, Dr Pancham Kumar ${ }^{2}$, Dr Ashok Garg ${ }^{3}$ \\ ${ }^{1}$ Junior Resident, ${ }^{2}$ Assistant Professor, ${ }^{3}$ Junior Resident \\ ${ }^{2,3}$ Department of Pediatrics, Indira Gandhi Medical College, Shimla- 171001, Himachal Pradesh, India \\ Corresponding Author \\ Dr Sandhya Chauhan
}

Junior Resident, Department of Dermatology, Venereology and Leprosy, Indira Gandhi Medical College,

Shimla- 171001, Himachal Pradesh, India

Email: drsandhya069@gmail.com, Mobile no.9817059771,9459373371

\begin{abstract}
A wide range of drugs have been implicated in the development of cardiac arrhythmias. Amongst them are the rarely reported drugs like hydroxyzine and some other antihistamines. Hydroxyzine induced cardiac arrhythmias oftenly occur with drug overdosage in pediatric population. Paroxysmal supraventricular tachycardia (PSVT) is a type of tachyarrhythmia rarely suspected in neonates secondary to hydroxyzine. Here we report twenty seven day old baby with life threatening PSVT secondary to hydroxyzine. It was the development of generalised rash on third day of viral illness which forced the parents to give over the counter (OTC) antiallergic syrup in view of drug reaction. After $3^{\text {rd }}$ dose of this (hydroxyzine), syrup a sick baby was admitted with unstable vitals, compromised cardiopulmonary functions with ECG evidence of PSVT. Baby was managed with adenosine injection and supportive measures. A detailed workup revealed the possibility of hydroxyzine induced PSVT after exclusion of all other causes. Clinician must be aware of drug induced arrhythmias in neonates, because fatalities can be avoided by early treatment along with weaning of culprit drug.
\end{abstract}

Key Words: PSVT, Hydroxyzine, Pediatric, Cardiac arrhythmia.

\section{INTRODUCTION}

Hydroxyzine is a potent H1antihistamine, commonly used in the pediatric population for a variety of skin allergies and pruritic disorders. Commonly reported side effects of hydroxyzine are; somnolence, impaired cognitive function and paradoxical hyperexcitability in pediatric patients. [1] Inappropriate dosage, pre-existing cardiac disease, dyselectrolytemia and concomitant arrhythmogenic drug intake are certain risk factors where cardiac arrhythmia may be precipitated. ${ }^{[2]}$
Reports of hydroxyzine induced PSVT are extremely sparse among pediatric population. Here we report a first case of hydroxyzine induced PSVT in twenty seven day old baby (neonate), although we could find only two more case reports of cardiac arrhythmia secondary to hydroxyzine in chidren (>1 year). ${ }^{[3,4]}$ As safety profile of Hydroxyzine has not been established in infants, a very cautious use is recommended with appropriate dosage and schedule. 


\section{CASE REPORT}

A sick looking, twenty seven days old male baby was brought in the emergency with complaints of fast breathing and sudden deterioration of general condition. Parents revealed that baby was on syrup paracetamol for viral illness from last 4 days. It was the development of generalised rash on third day of illness which forced the parents to give over the counter (OTC) hydroxyzine in view of drug reaction. They started hydroxyzine $2.5 \mathrm{ml}$ $(5 \mathrm{mg})$ three times daily doses after consulting a local chemist. After $3^{\text {rd }}$ dose (Total cumulative dose $=15 \mathrm{mg}$ ) of hydroxyzine baby's condition deteriorated suddenly and progressed into fast and difficult breathing over a period of 5-10 hours. At the time of presentation baby was in respiratory distress with RR- 72/minute, HR: 280-300/minute, dusky lips, oxygen saturation- $68 \%$. Baby was afebrile with body weight of $3.5 \mathrm{~kg}$. The peripheral pulses were palpable and blood pressure recorded as $65 / 30 \mathrm{mmHg}$ with MAP of $43 \mathrm{mmHg}$. Systemic examination revealed the feature of congestive cardiac failure (CCF) in the form of crepitation in bilateral infrascapular, infraaxillary areas and hepatomegaly with liver span of $9.5 \mathrm{~cm}$. ECG showed narrow complex tachycardia (HR of $290 /$ minute) with no beat to beat variation in rhythm, these findings confirmed the diagnosis of PSVT (Figure1).



Fig: 1 ECG Showing PSVT

Supportive measures were started immediately and a fast bolus of intravenous adenosine in a dose of $0.1 \mathrm{mg} / \mathrm{kg}$ was administered. HR dropped to 130-140/minute on ECG (Figure 2), further improvement was observed in respiratory rate, oxygen saturation and activity.



Fig: 2 ECG after adenosine injection (PSVT aborted).

$\mathrm{CCF}$ and respiratory problems improved slowly and baby was weaned of all supportive measures over next 12 hours. Subsequently ECG examination, echocardiographic, electrophysiological and electrolyte studies were performed. No abnormalities were appreciated on detailed workup, so a possibility of hydroxyzine induced
PSVT was kept. At discharge, parents were educated about the symptoms of PSVT and also advised not to give OTC medication without specialist consultation. Baby is doing well as per monthly follow up examination for last four months. 


\section{DISCUSSION}

PSVT is a rare neonatal tachyarrhythmia, defined by sustained, accelerated, non-sinus rhythm arising from or above the atrioventricular (AV) node. The prevalence in childhood ranges from one in 25,000 to one in 250 individuals. ${ }^{[5]}$ Fever, emotional upset, nicotine, caffeine and certain drugs are common precipitating factors. Drugs with anticholinergic properties (tricyclic antidepressants and first-generation antihistamines) can cause tachycardia through their effect on the parasympathetic nervous system- by blocking vagal stimulation. ${ }^{[2]}$ Hydroxyzine is a most potent $1^{\text {st }}$ generation histamine (H1) receptor antagonist with long biological action. It has versatile uses in pediatric dermatosis due to low toxicity and favourable pharmacokinetic properties like rapid absorption, plasma half-life of seven hours in children and sustained suppression of pruritus for 1-24 hours. ${ }^{[1]}$ Cardiac toxicity is a very rarely reported adverse event with hydroxyzine. Literature search showed one case report of sinus tachycardia due to accidental ingestion of hydroxyzine in a 13 -month-old child. ${ }^{[4]}$ Wong et al reported another case of supraventricular tachycardia in a nine-year-old child. ${ }^{[3]}$ This is the third case of hydroxyzine induced cardiac toxicity in pediatric age group although it is first reported case of neonatal PSVT. We suppose that it was the overdose of hydroxyzine which resulted in the adverse event, though it remained unproven.

PSVT can have variable clinical manifestations including irritability, poor feeding, rapid breathing, mottling and cyanosis in infancy/neonatal period. The clinical manifestations in neonatal period can be easily confused with sepsis, or respiratory distress secondary to pulmonary disease and same was suspected in our case. Recordable signs include tachycardia, tachypnea, prolonged CRT, weak pulses, cool extremities, diaphoretic, pale, mottled skin, hypotension, shock and features of congestive cardiac failure. ${ }^{[6]}$ Characteristic features of PSVT on ECG are: heart rate of greater than 220 beats per minute in infants (> 180 beats per minute in children/adolescents). The QRS complexes' are narrow $(<.08-.12$ seconds) and $\mathrm{P}$ wave may not be identifiable. The rate is regular with no beat-to-beat variation in rhythm. ECHO is advisable to rule out structural cardiac disease and electrophysiological studies are done to map accessory pathway. ${ }^{[6]}$

PSVT can be terminated by simple vagal manoeuvres like; application of ice packs over face, valsalva manoeuvre and carotid sinus massage under ECG monitoring. In unstable child these measures can be tried while making preparation for pharmacology or electro-cardio version. Drug of choice for acute attack of PSVT is intravenous adenosine with initial dose of 50$100 \mathrm{mcg} / \mathrm{kg}$. Sinus rhythm is restored in $90 \%$ cases but prior to give adenosine blood pressure should be stable. ${ }^{[7]}$ Due to the extremely short half-life of 10 to 15 seconds, adenosine is administered as a rapid bolus over 1 to 2 seconds and followed by rapid flush of 2-3 $\mathrm{ml}$ of normal saline. If cardiac rhythm fails to restore, a double dose can be repeated after 2- minutes. Adenosine can be given to a maximum dose of $300 \mathrm{mcg} / \mathrm{kg}$ or increments of $50 \mathrm{mcg} / \mathrm{kg}$ is done at intervals of 2 minutes until sinus rhythm is restored. ${ }^{[8]}$ Other drugs which can be used for PSVT are verapamil, propranolol and flecainide. If pharmacological conversion fails or baby is haemodynamically unstable, electrical cardioversion (synchronised cardioversion: 0.5-1.0 Joules $/ \mathrm{kg}$ ) is the treatment of choice. ${ }^{[5]}$ Digoxin, beta-blocker, flecainide and amiodarone are used as the long term/periodic pharmacotherapy. Catheter ablation and surgery are the other treatment modalities. Education of the parents regarding the precipitating factors for PSVT and avoidance of these factors is very important.

\section{CONCLUSION}

Hydroxyzine is used widely in pediatric population but its safety has not been established in infancy. In all cases of cardiac arrhythmias, detailed drug history is of utmost important especially about the dosage in children. PSVT is life threatening event in children which can occur even with safe considered drugs like hydroxyzine. Drug induced PSVT can have excellent prognosis 
if culprit drug is stopped immediately. Further early diagnosis with ECG and timely termination of the event with pharmacotherapy or electrocardio version can avoid fatalities.

\section{REFERENCES}

1. Simons FER, Simons KJ. H1 Antihistamines Current Status and Future Directions. WAO Journal 2008;145-155.

2. Wooten J. Drug-induced arrhythmias. RN 2002;1:37.

3. Wong AR, Rasool AHG. HydroxyzineInduced Supraventricular Tachycardia in a Nine-year-old Child. Singapore Med J 2004;45:91.

4. Magera BE, Betlach CJ, Sweatt AP, Derrick CW. Hydroxyzine intoxication in a 13 month old child. Pediatrics 1981;67:280-283.

5. Atkins DL. Advances in resuscitation: diagnosis and management of supraventricular tachycardia in children. Clin Ped Emerg Med 2001;2:107-111.

6. Garson A, Gillette PC, McNamara DG. Supraventricular tachycardia in children: clinical features, response to treatment, and long-term follow-up in 217 patients. J Pediatr 1981;98:875-882.

7. Ralston MA, Knilans TK, Hannon DW, Daniels SR. Use of adenosine for diagnosis and treatment of tachyarrhythmias in pediatric patients. J Pediatr 1994;124:139-143.

8. Till J, Shinebourne EA, Rigby ML, Clarke B, Ward DE, Rowland E. Efficacy and safety of adenosine in the treatment of supraventricular tachycardia in infants and children. Br Heart J 1989;62:204-211. 